\title{
Clinical Medicine Insights: Cardiology
}

\section{Ventricular Tachycardia in Pregnant Patients}

\author{
Rouslan Kotchetkov ${ }^{1}$, Ameen Patel ${ }^{1}$ and Omid Salehian ${ }^{2}$ \\ ${ }^{1}$ Department of Medicine, ${ }^{2}$ Division of Cardiology, McMaster University, Hamilton, Ontario, Canada. \\ Email: salehian@hhsc.ca
}

\begin{abstract}
Ventricular tachycardia although not common, can occasionally complicate pregnancy. Its presence may indicate an underlying cardiac structural abnormality, or undiagnosed congenital arrhythmic disease. However, some pregnant patients with ventricular tachycardia have structurally normal hearts. Two cases of ventricular tachycardia in pregnant patients with structurally normal hearts are presented and an approach to diagnosis and management of such patients are discussed.
\end{abstract}

Keywords: ventricular tachycardia, VT, pregnancy

Clinical Medicine Insights: Cardiology 2010:4 39-44

This article is available from http://www.la-press.com.

(C) the author(s), publisher and licensee Libertas Academica Ltd.

This is an open access article. Unrestricted non-commercial use is permitted provided the original work is properly cited. 


\section{Case 1}

A 33-year-old woman, (gravida 3, para 2), at 34 weeks of gestation, presented with episodes oflightheadedness, and dyspnea. She was asymptomatic until 18 weeks of gestation, when she started having episodes of palpitations associated with presyncope. Initially, the episodes were infrequent (less than once per day) but a gradual increase in frequency and severity was noted by the patient. One month prior to presentation, she noted episodes of paroxysmal nocturnal dyspnea, but no dyspnea on exertion, chest pain, diaphoresis, pedal edema, orthopnea, or neurological symptoms.

She had no significant past medical history and had not had complications relating to this pregnancy. All prior pregnancies had progressed to full term without similar symptoms or complications, and resulted in spontaneous vaginal deliveries of healthy newborns. The patient was using no medications except multivitamins. There was no history of smoking, alcohol consumption or illicit drug use.

Physical examination revealed an afebrile patient with a regular pulse of 80 beats per minute, blood pressure 110/70 $\mathrm{mmHg}$, and an oxygen saturation of $100 \%$ on room air. The examination was normal except for mild non-pitting edema and fine bibasilar crackles.
Laboratory investigations revealed normal complete blood count, electrolytes, renal function, cardiac markers and TSH. Cardiac monitoring showed multiple episodes of monomorphic, non-sustained ventricular tachycardia (VT) with left bundle branch morphology, consistent with VT originating from the right ventricular outflow tract (Fig. 1). These episodes occurred both at rest and with ambulation. Urgent two-dimensional echocardiogram showed normal left ventricular size and wall thickness with no segmental wall motion abnormalities and normal systolic and diastolic function with a left ventricular ejection fraction of $57 \%$. There were no valvular abnormalities. A 12-lead electrocardiogram showed sinus rhythm with average rate of 80 beats $/ \mathrm{min}$ with a normal axis, PR-interval, and QRS duration with no evidence of chamber enlargement or infarction. The clinical working diagnosis was pregnancy-related VT of right ventricular outflow tract origin.

\section{Case 2}

A 24 year woman (gravida 4, para 2) presented to her local emergency department at 24 weeks gestation because of shortness of breath, cough, and chest tightness developing over the previous day. There were no other cardiorespiratory symptoms. Her pregnancy had been progressing normally.

16:46

SPEED $=25.00 \mathrm{MM} / \mathrm{S}$
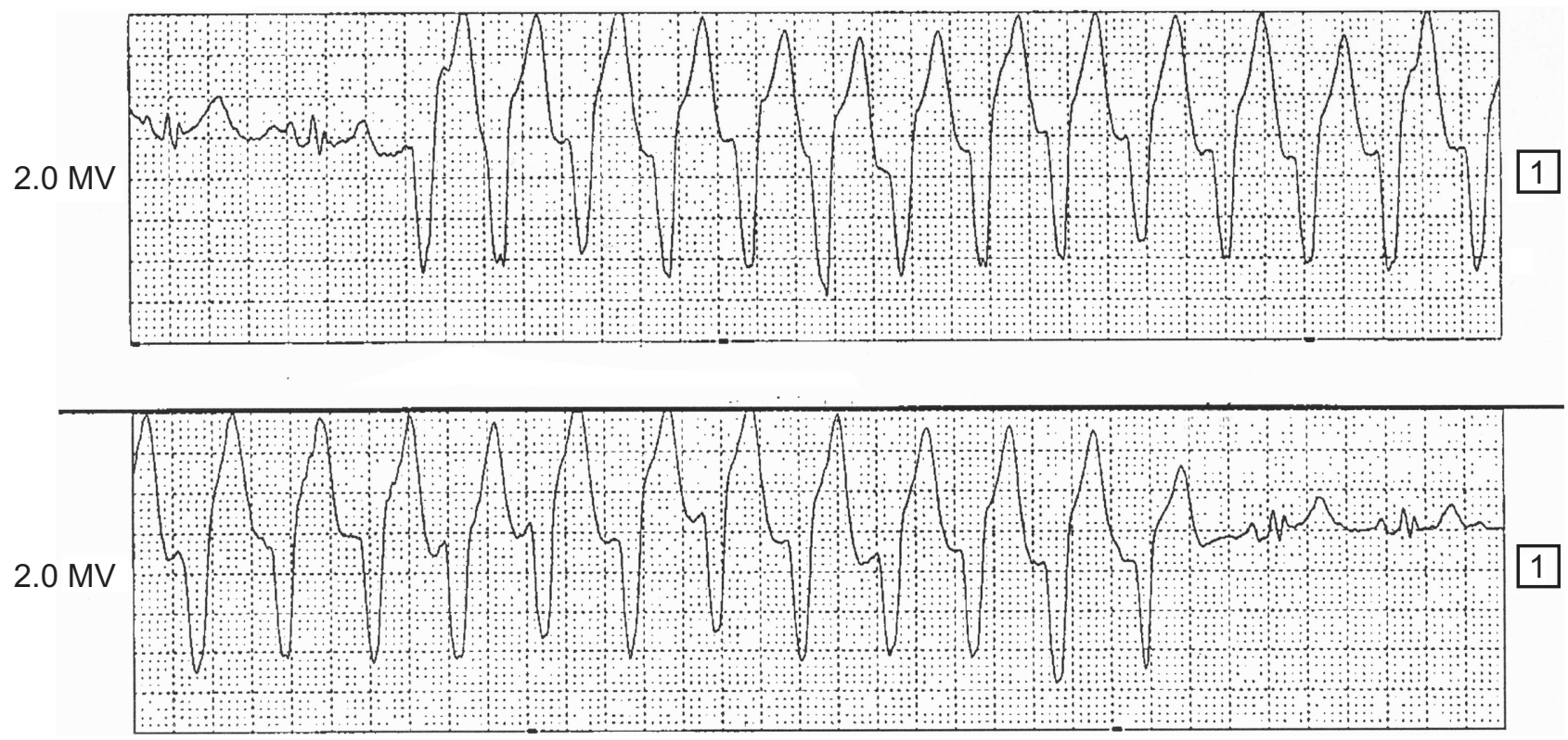

Figure 1. Telemetry cardiac rhythm recording of Patient 1 (lead I), showing wide complex tachycardia at a rate of 160 bpm. 
She had a history of mild asthma but had not had asthma-related symptoms for several years and had never had an asthma exacerbation requiring inpatient or emergency department care. Past medical history and family history were otherwise unremarkable. There had been no similar symptoms or medical complications in her previous three pregnancies, one of which had ended with spontaneous abortion and the other two with term delivery of healthy children.

The patient was using no medications. She lived with her husband and two children. She smoked onehalf to one pack of cigarettes per day but did not drink alcohol or use recreational drugs.

Physical examination revealed an afebrile patient with a regular pulse of 78 beats per minute, blood pressure 100/60 $\mathrm{mmHg}$, and an oxygen saturation of $96 \%$ on room air. On respiratory exam she was slightly tachypneic and had expiratory wheezes. The rest of the examination was normal except for mild non-pitting peripheral edema.

Laboratory investigations revealed normal complete blood count, electrolytes, renal function, cardiac markers, and TSH. Cardiac monitoring showed ventricular trigeminy. The patient was diagnosed with an asthma exacerbation and treated with nebulized salbutamol $2.5 \mathrm{mg}$, which relieved her symptoms. Shortly thereafter, she developed runs of asymptomatic nonsustained VT with a left bundle branch block pattern (Fig. 2). Her shortness of breath recurred later in the day. Nebulized salbutamol $5 \mathrm{mg}$ was once again administered, whereupon the patient developed sustained runs of VT of similar morphology. Intravenous lidocaine and magnesium sulfate were administered, leading to resolution of the ventricular arrhythmia. The patient was transferred to the coronary care unit of our hospital. No further salbutamol was administered, and two further days of electrocardiographic monitoring showed only occasional PVCs. Twelve lead electrocardiogram showed sinus tachycardia at 102 beats per minute with occasional premature ventricular beats, normal corrected QT interval and no evidence of ischemia or chamber enlargement. Echocardiography showed normal left ventricular size with normal systolic function with an ejection fraction of $57 \%$, and no valvular abnormalities.

Given the absence of structural heart disease and risk factors for coronary disease or arrhythmia, the clinical diagnosis was catecholamine-sensitive pregnancy-related VT of right ventricular outflow tract origin. The temporal relationship to salbutamol use suggests that this agent may have precipitated VT.

\section{Discussion}

Ventricular tachycardias (VT) may be seen in pregnancy and can manifest as a new onset arrhythmia or be exacerbated by pregnancy and can cause concern for the well-being of both the mother and the fetus. Ventricular tachycardia may manifest at any time during pregnancy. A study of 11 pregnant women who experienced a new onset of VT during pregnancy showed that onset was distributed equally over the three trimesters and disappeared completely during the postpartum period. ${ }^{1}$ The characteristics and underlying mechanisms of new-onset VT during pregnancy have not been adequately investigated. Although the majority of VTs that occur during the pregnancy are benign, in women with structural heart disease, arrhythmias (especially those of ventricular origin) is one of the five independent predictors of having an adverse outcome during pregnancy. ${ }^{2,3}$

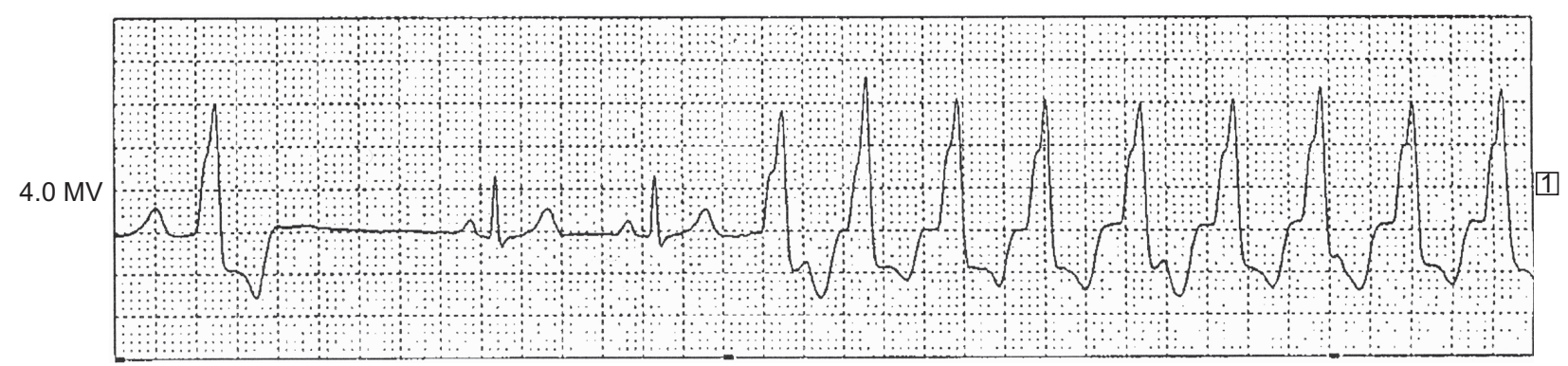

Figure 2. Telemetry cardiac rhythm recording of Patient 2 (lead II), showing wide complex tachycardia at a rate of $150 \mathrm{bpm}$. 
Peripartum cardiomyopathy (PPCM) needs to be considered in women presenting with VT in the last month of pregnancy. ${ }^{4,5}$ The criteria for the diagnosis of PPCM include: development of congestive heart failure secondary to left ventricular systolic dysfunction in the last month of pregnancy or within 5 months after delivery, absence of preexisting cardiac dysfunction, and absence of identifiable cause of heart failure and LV systolic dysfunction demonstrated by classic echocardiographic criteria. ${ }^{6}$ Hence in women presenting with VT in the last month of pregnancy, an urgent echocardiographic study can assist in establishing a diagnosis of PPCM.

Women with certain types of congenital heart disease (CHD) either repaired or not, are particularly vulnerable to VT. For example, patients with previous surgeries for correction of tetralogy of Fallot may experience VT arising from the right ventricular outflow tract as a result of right ventricular volume overload due to decades of severe pulmonary regurgitation as a consequence of patch augmentation of the right ventricular outflow tract at the time of original corrective surgery. ${ }^{7}$ A study of 28 female patients with repaired CHD demonstrated women with CHD had a significantly higher incidence of tachyarrhythmia during pregnancy and in the postpartum period compared to controls. ${ }^{8}$

Congenital long QT syndrome (LQTS) is a hereditary disorder of cardiac ion channels causing abnormal electrical activation of the heart. ${ }^{9}$ Women with congenital LQTS may experience life-threatening polymorphic VT; however recent reports indicates that their incidence appears to be significantly increased during the postpartum period. ${ }^{10,11}$ Patients with congenital LQTS are particularly vulnerable to adverse arrhythmic effects of electrolyte depletion (further prolongation of QT interval) a possible complication in some pregnant women with hyperemesis. Cautious use of mediations that may further prolong the QT interval is necessary in these individuals to avoid precipitation of polymorphic VT. In addition to cardiac diseases, several systemic non-cardiac disorders, including severe electrolyte derangements, thyroid function abnormalities, pulmonary embolism, anemia, and drug overdose may present with ventricular arrhythmias.

There are few reports of new onset VT during pregnancy in absence of structural heart disease..$^{12,13}$
Increased sympathetic activity, as well as physiological changes associated with normal pregnancy such as increased heart rate, decreased peripheral resistance, increased stroke volume and psychological stresses are thought to be the most common precipitants of VT in pregnant women with a structurally normal hearts. ${ }^{14,15}$ The majority of outflow tract VT in structurally normal hearts originate from the Right Ventricular Outflow Tract (RVOT) just below the pulmonary valve. This is also the most common focus of initiation of pregnancy-related idiopathic VT and usually does not deteriorate to an unstable rhythm. It will often manifest as ectopic beats, bigeminal rhythms or short runs of non-sustained VT. Ventricular tachycardia originating from the RVOT is associated with states of hormonal fluctuation. Increased norepinephrine concentration in the myocardial synaptic cleft has been suggested as a potential mechanism for this arrhythmia. ${ }^{16}$ Only $10 \%$ of VT originates from the Left Ventricular Outflow Tract (LVOT). ${ }^{17}$

To make a diagnosis, a 12-lead ECG should be recorded ideally during the arrhythmia, although this can be difficult. A typical resting ECG of a pregnant woman may show an increased heart rate with decreased PR, QRS and QT intervals, but there is usually no significant change in the amplitudes of $\mathrm{P}$ and $\mathrm{T}$-waves and QRS-complex. ${ }^{15}$ Premature atrial and ventricular beats as well as Q wave and inverted $\mathrm{T}$ waves in the inferior leads can also be seen during pregnancy. ${ }^{18}$

On ECG, RVOT VT appears as wide QRS complex tachycardia with left bundle brunch block (LBBB) morphology and an inferior axis. ${ }^{17,19}$ Two phenotypic forms of RVOT VT can occur, non-sustained monomorphic VT and paroxysmal sustained VT. ${ }^{20}$ In contrast, LVOT VT morphology depends on the site of origin with either LBBB and early precordial transition in leads $\mathrm{V}_{1-2}$, or RBBB pattern in $\mathrm{V}_{1}$ with broad monophasic $\mathrm{R}$ in the precordial leads. In addition, the ECG in patients with VT originating in the LVOT will show R:S amplitude ratio of $30 \%$ or more or an $\mathrm{R}: \mathrm{QRS}$ duration ratio of $50 \%$ in leads $\mathrm{V}_{1-2}{ }^{21}$

A routine 24 to 48 hour-Holter monitor is helpful in capturing frequently occurring paroxysmal arrhythmias. To elicit symptoms, an exercise ECG can be reasonably carried out during pregnancy in absence of obstetrical contraindications for exercise. Alternatively, a pharmacological challenge may 
provide important diagnostic information. Adenosine has been safely used in pregnancy. No fetal adverse events are reported for adenosine, except for one case of transient fetal bradycardia. ${ }^{22}$

The first step in acute management of VT in pregnancy to determine the hemodynamic stability of the pregnant woman. If the woman is unstable or there is evidence of significant fetal compromise which is thought to be related to the VT, DC cardioversion with 50-100 J should be given immediately. DC shocks can be repeated at higher levels of energy (100-360 $\mathrm{J})$ if indicated. The risk for the fetus is minimal for all stages of pregnancy, because the amount of current reaching the fetus is small. ${ }^{23}$ In stable VT, an accurate diagnosis of the type of arrhythmia should be made with a twelve-lead ECG prior to any intervention. Invasive electrophysiological studies are rarely required during pregnancy, as the arrhythmias can be effectively managed pharmacologically.

Conservative medical treatment of VT arising during pregnancy is indicated in any patient with sustained VT as long as the patient is hemodynamically stable. ${ }^{14}$ If possible drug therapy should be avoided during the first trimester and drugs with the longest records of safety should be used as the first-line therapies. ${ }^{24}$ The literature safety data has examined digoxin, adenosine, flecainide, procainamide, propranolol, propafenone, quinidine, sotalol and verapamil, however; the experience is limited to single or small case series. In stable patients with sustained VT acute therapy can be initiated with either intravenous procainamide, which is safe, well-tolerated and is not associated with teratogenicity. Alternatively Lidocaine can also be used to treat stable VT. ${ }^{14}$ Idiopathic VT originating from the RVOT and with a LBBB morphology responds well to beta-blockers, while idiopathic LVOT VT with RBBB morphology generally responds well to verapamil. Given the sensitivity of majority of VTs in pregnancy to catecholamines, a cardioselective beta-blocker, in particular metoprolol, in the absence of contraindications is considered first line therapy. ${ }^{12}$

\section{Return to the Cases}

In our cases, further investigations did not reveal a secondary cause of the patients' arrhythmias. Based on the ECGs (QRS morphology of a left bundle branch block pattern with an inferior axis, and clinical findings) both patients were diagnosed with idiopathic VT originating from the RVOT.

Management with oral metoprolol at 25 and $12.5 \mathrm{mg}$ twice a day was initiated. The lower dose for the second patient was chosen because of suspected asthma exacerbation; however the dose was slowly increased to $50 \mathrm{mg}$ PO every 12 hours. Both patients tolerated treatment well and had no further documented episodes of VT on long-term cardiac monitoring. Patient 1 was scheduled for elective $\mathrm{C}$-section at a high risk obstetric center and was discharged home. On the follow-up she did not have further episodes of palpitations or presyncopal/syncopal episodes. Patient 2 had a spontaneous vaginal delivery at 36 weeks and continued to be on metoprolol after discharge.

\section{Conclusion}

Pregnant patients may present with ventricular tachycardia during any trimester Careful and timely clinical, electrocardiographic, as well as echocardiographic assessment help identify those individuals with structural cardiac abnormalities such as congenital heart disease or peripartum cardiomyopathy, as well as those with long QT syndrome who will need specific management beyond therapy for the arrhythmia. In those stable patients with structurally normal hearts, identification of the location of origin of tachycardia will help in choice of appropriate medical therapy.

\section{Disclosures}

This manuscript has been read and approved by all authors. This paper is unique and is not under consideration by any other publication and has not been published elsewhere. The authors and peer reviewers of this paper report no conflicts of interest. The authors confirm that they have permission to reproduce any copyrighted material.

\section{References}

1. Nakagawa M, Katou S, Ichinose $M$, et al. Characteristics of new-onset ventricular arrhythmias in pregnancy. $J$ Electrocardiol. 2004 January;37(1): 47-53.

2. Siu SC, Sermer M, Harrison DA, et al. Risk and predictors for pregnancyrelated complications in women with heart disease. Circulation. 1997 November 4;96(9):2789-94.

3. Siu SC, Sermer M, Colman JM, et al. Prospective multicenter study of pregnancy outcomes in women with heart disease. Circulation. 2001 July 31;104(5):515-21.

4. Sliwa K, Fett J, Elkayam U. Peripartum cardiomyopathy. Lancet. 2006 August 19;368(9536):687-93. 
5. Abboud J, Murad Y, Chen-Scarabelli C, Saravolatz L, Scarabelli TM. Peripartum cardiomyopathy: a comprehensive review. Int J Cardiol. 2007 June 12;118(3):295-303.

6. Pearson GD, Veille JC, Rahimtoola S, et al. Peripartum cardiomyopathy: National Heart, Lung, and Blood Institute and Office of Rare Diseases (National Institutes of Health) workshop recommendations and review. JAMA. 2000 March 1;283(9):1183-8.

7. Adamson DL, Nelson-Piercy C. Managing palpitations and arrhythmias during pregnancy. Heart. 2007 December;93(12):1630-6.

8. Niwa K, Tateno S, Akagi T, et al. Arrhythmia and reduced heart rate variability during pregnancy in women with congenital heart disease and previous reparative surgery. Int J Cardiol. 2007 November 15;122(2):143-8.

9. Engelstein ED. Long QT syndrome: a preventable cause of sudden death in women. Curr Womens Health Rep. 2003 April;3(2):126-34.

10. Wolbrette D. Treatment of arrhythmias during pregnancy. Curr Womens Health Rep. 2003 April;3(2):135-9.

11. Seth R, Moss AJ, McNitt S, et al. Long QT syndrome and pregnancy. J Am Coll Cardiol. 2007 March 13;49(10):1092-8.

12. BrodskyM,Doria R,Allen B, Sato D, Thomas G, Sada M.New-onsetventricular tachycardia during pregnancy. Am Heart J. 1992 April;123(4 Pt 1):933-41.

13. Wolbrette D, Patel H. Arrhythmias and women. Curr Opin Cardiol. 1999 January; 14(1):36-43.

14. Trappe HJ. Acute therapy of maternal and fetal arrhythmias during pregnancy. J Intensive Care Med. 2006 September;21(5):305-15.

15. Gowda RM, Khan IA, Mehta NJ, Vasavada BC, Sacchi TJ. Cardiac arrhythmias in pregnancy: clinical and therapeutic considerations. Int $J$ Cardiol. 2003 April;88(2-3):129-33.
16. Schafers MA, Wichter T, Schafers KP, et al. Pulmonary beta adrenoceptor density in arrhythmogenic right ventricular cardiomyopathy and idiopathic tachycardia. Basic Res Cardiol. 2001 February;96(1):91-7.

17. Lerman BB, Stein KM, Markowitz SM. Mechanisms of idiopathic left ventricular tachycardia. J Cardiovasc Electrophysiol. 1997 May;8(5):571-83.

18. Shotan A, Ostrzega E, Mehra A, Johnson JV, Elkayam U. Incidence of arrhythmias in normal pregnancy and relation to palpitations, dizziness, and syncope. Am J Cardiol. 1997 April 15;79(8):1061-4.

19. Buxton AE, Marchlinski FE, Doherty JU, et al. Repetitive, monomorphic ventricular tachycardia: clinical and electrophysiologic characteristics in patients with and patients without organic heart disease. Am J Cardiol. 1984 November 1;54(8):997-1002.

20. Nathani P, Shetty S, Lokhandwala Y. Ventricular tachycardia in structurally normal hearts: recognition and management. J Assoc Physicians India. 2007April;55 Suppl:33-8.

21. Ouyang F, Fotuhi P, Ho SY, et al. Repetitive monomorphic ventricular tachycardia originating from the aortic sinus cusp: electrocardiographic characterization for guiding catheter ablation. J Am Coll Cardiol. 2002 February 6;39(3):500-8.

22. Dunn JS Jr, Brost BC. Fet al bradycardia after IV adenosine for maternal PSVT. Am J Emerg Med. 2000 March;18(2):234-5.

23. Finlay AY, Edmunds VDC. cardioversion in pregnancy. Br J Clin Pract. 1979 March;33(3):88-94.

24. Joglar JA, Page RL. Antiarrhythmic drugs in pregnancy. Curr Opin Cardiol. 2001 January; 16(1):40-5.

\section{Publish with Libertas Academica and every scientist working in your field can read your article}

"I would like to say that this is the most author-friendly editing process I have experienced in over 150 publications. Thank you most sincerely."

"The communication between your staff and me has been terrific. Whenever progress is made with the manuscript, I receive notice. Quite honestly, l've never had such complete communication with a journal."

" $L A$ is different, and hopefully represents a kind of scientific publication machinery that removes the hurdles from free flow of scientific thought."

Your paper will be:

- $\quad$ Available to your entire community free of charge

- $\quad$ Fairly and quickly peer reviewed

- Yours! You retain copyright

http://www.la-press.com 